\title{
Cuidado transcultural de Leininger na perspectiva dos programas de pós-graduação em enfermagem: revisão integrativa
}

\author{
Leininger's transcultural care from the perspective of postgraduate programs \\ in nursing: integrative review
}

\author{
Luizita Henckemaier ${ }^{1}$, Josiane Steil Siewert ${ }^{2}$, Lilian Cristiana Júlio Tonnera ${ }^{3}$, Angela Maria \\ Alvarez $^{4}$, Betina Hörner Schlindwein Meirelles ${ }^{5}$, Rosane Gonçalves Nitschke ${ }^{6}$
}

\section{RESUMO}

Objetivo: Identificar a aplicação da Teoria da Diversidade e Universalidade do Cuidado Cultural de Madeleine Leininger, nas produções científicas em Programas de Pós-Graduação em Enfermagem nas regiões sul e sudeste do Brasil, no período de 2005 a 2010.

Materiais e Métodos: Trata-se de revisão integrativa da literatura. A busca foi realizada no catálogo da ABEN, totalizando 17 produções, destas, 16 dissertações de mestrado e 01 tese de doutorado.

Resultados: Verificou-se que a teoria de Leininger foi utilizada parcialmente, tanto como metodologia para pesquisa, como também como forma de sistematizar o cuidado ou apenas como referencial teórico para a discussão dos dados. A utilização de diversas técnicas para coleta e análise dos dados foi detectada durante a leitura criteriosa das produções.

Conclusão: Percebe-se, portanto, a necessidade de ampliação da utilização da teoria de Leininger. Desta forma, será possível sentir as melhorias no processo de cuidar, sendo necessário repensar o entendimento do que é o cuidado, como ele deve ser prestado e qual o olhar dado pelas equipes de enfermagem neste âmbito.

Palavras-chave: pesquisa em enfermagem; cultura; teoria de enfermagem.

\section{ABSTRACT}

Objective: To identify the application of Leiningerś Theory of Diversity and Universality of the Cultural Care in the post graduate programs in nursing in southern and southeastern Brazil, from 2005 to 2010.

Materials and Methods: This is an integrative review. The search was realized in the catalog of ABEN, totaling 17 productions, in which 16 were dissertations and 01 was a thesis.

Results: It was found that Leininger's theory was partially used in these productions, as a methodology for research, as a systematic care and as a theoretical framework for data discussion. The use of various techniques for data collection and analyses was also detected after careful reading of the productions included.

Conclusions: It is observed the need to expand the use of the theory of Leininger. Thus, it is possible to achieve improvements in the care process, being also necessary to rethink the understanding of what is care, how it should be provided and what is the look given by the nursing staff in this area.

Keywords: nursing research; culture; nursing theory.

\footnotetext{
${ }^{1}$ Enfermeira. Doutoranda em Filosofia da Enfermagem. Hospital Universitário Professor Polydoro Ernani de São Thiago. Programa de Pós-Graduação em Enfermagem. Núcleo de Pesquisa e Estudos sobre Enfermagem, Quotidiano, Imaginário, Saúde e Família de Santa Catarina (NUPEQUISFAM-SC). Universidade Federal de Santa Catarina (UFSC).

${ }^{2}$ Enfermeira. Mestre em Filosofia da Enfermagem. Instituto Federal de Santa Catarina. Grupo de Estudos sobre Cuidado de Saúde de Pessoas Idosas (GESPI). UFSC.

${ }^{3}$ Enfermeira. Mestre em Filosofia da Enfermagem. Secretaria Estadual de Saúde de Santa Catarina. Núcleo de Estudos e Assistência em Enfermagem e Saúde a Pessoas com Doenças Crônicas (NUCRON). UFSC.

${ }^{4}$ Enfermeira. Doutora em Filosofia da Enfermagem. Programa de Pós-Graduação em Enfermagem. GESPI. UFSC.

${ }^{5}$ Enfermeira. Doutora em Filosofia da Enfermagem. Programa de Pós-Graduação em Enfermagem. NUCRON. UFSC.

${ }^{6}$ Enfermeira. Doutora em Filosofia da Enfermagem. Programa de Pós-Graduação em Enfermagem. NUPEQUISFAM-SC. UFSC.
} 


\section{INTRODUÇÃO}

A prática de cuidado em saúde pode ser considerada como uma ação que se inicia dentro do ambiente familiar, a qual é aplicada de diversas formas aos diferentes componentes que a constituem, frente às necessidades de cuidar que estes possam ser submetidos ${ }^{1}$.

No que concerne o campo das profissões voltadas para a assistência à saúde, a enfermagem mantém-se hoje como uma profissão que tem como preocupação o cuidado dispensado ao ser humano, nos mais diferentes ambientes e contextos de cuidado em saúde, envolvendo suas distintas culturas. Uma das maneiras para se efetivar essa preocupação da enfermagem é o de "estudar as teorias específicas", o que contribuirá para "uma prática mais eficiente e resolutiva" ${ }^{2: 43}$. Vale ressaltar que o fato de estudar as teorias, não significa aceitá-las em sua totalidade, mas sim "utilizar um corpo de conhecimento próprio", adaptando aos conteúdos teóricos, de acordo com as afinidades da teoria com a prática $^{2: 44}$.

Ao abordar as teorias de enfermagem, estas se destacam como "expressões dos valores e crenças profissionais, apontando a referência que irá fundamentar o trabalho e a relação terapêutica". Estas, por sua vez, possuem uma abrangência da "visão sobre o significado do sofrimento para as pessoas, a diversidade do cuidado humano, a unicidade da pessoa, sua expressão física, verbal ou espiritual”2:166 .

Neste âmbito, vemos se destacar a Teoria da Diversidade e Universalidade do Cuidado Cultural de Madeleine Leininger, a qual é apontada como uma teoria que tem como grande preocupação o cuidado baseado nas crenças, valores e atitudes de cada pessoa e cultura ${ }^{3}$.

A importância de pesquisar este tema está pautada no interesse em proporcionar aos enfermeiros, o conhecimento acerca do tema e como este tem sido abordado nas práticas de cuidado em saúde em enfermagem, assim como, permitir realizar trocas de conhecimentos que possam oportunizar exemplos práticos de cuidado fundamentado no desenvolvimento teórico das atitudes, crenças e valores do ser humano. Pesquisar as diversas formas de cuidados existentes sob a ótica do Cuidado Transcultural de Leininger proporcionou-nos mergulhar no universo destas, os quais servem de estímulos para a adoção de uma teoria com um cuidado fundamentado cientificamente para servir de sustentação as práticas de cuidados prestados no dia a dia do enfermeiro nos mais variados contextos de saúde, ou seja, o contexto familiar, institucional e social.

Considerando as práticas de cuidado em enfermagem existentes baseado no conhecimento teórico, este estudo está pautado na seguinte questão norteadora: Como está sendo aplicada a Teoria do Cuidado Transcultural de Madeleine Leininger nas produções dos Programas de Pós-Graduação em Enfermagem das regiões sul e sudeste do Brasil?

Sendo assim, este estudo teve como objetivo: identificar a aplicação da Teoria da Diversidade e Universalidade do Cuidado Cultural, de Madeleine Leininger, nas produções científicas dos Programas de Pós-Graduação de Enfermagem das regiões sul e sudeste do Brasil, no período de 2005 a 2010.

\section{MATERIAIS E MÉTODOS}

Trata-se de uma revisão integrativa da literatura, que constou na elaboração e validação de um protocolo de pesquisa relacionado à coleta, análise e apresentação dos resultados, a qual se deu através da adaptação de seis critérios sugeridos por Mendes, Silveira, Galvão ${ }^{4}$.

O primeiro passo para a inclusão dos trabalhos, foi identificar as produções científicas do banco de teses e dissertações publicadas no Centro de Estudos e Pesquisas em Enfermagem (CEPEn) da Associação Brasileira de Enfermagem - Nacional (ABEn/N), durante os meses de novembro e dezembro de 2011. Assim, foram acessados os catálogos dos anos de 2005 a 2010, quando se buscou resgatar, através dos títulos e dos resumos as produções que trabalharam com cultura, cuidado transcultural, cuidado transcultural em enfermagem, etnoenfermagem e a teoria de Madeleine Leininger. A pesquisa foi feita através dos títulos e resumos, pois o banco de teses está em formato de catálogo, não permitindo realizar buscas através de descritores.

Para dar maior abrangência e aprofundamento às 
buscas, no segundo passo foram acessadas e lidas, na íntegra, as dissertações e teses no intuito de identificar a presença ou não do uso da teoria, e posterior exclusão das que não referiram o cuidado da Leininger em sua produção.

De acordo com o banco de dados da ABEn Nacional, foram encontradas 2037 produções, sendo 561 teses de doutorado e 1476 dissertações de mestrado. Para a préseleção, foram estabelecidos os seguintes critérios: trabalhos de dissertação ou tese, disponível na íntegra, ter sido concluído no período de 2005 a 2010, e ter utilizado a teoria de Leininger na íntegra ou de forma parcial. Procedeu-se a pré-seleção, resultando em 27 produções, das quais 05 foram excluídas por se tratarem da utilização de outras teorias baseadas na antropologia do cuidado e não na Teoria da Diversidade e Universalidade do Cuidado Cultural de Madeleine Leininger e 05, foram excluídas por não se encontrarem disponibilizadas por completo conforme critério de exclusão pré-estabelecido. Para estas, ainda foram feitas novas buscas nos sites das universidades de origem destas produções, bem como no site de domínio público, com a finalidade de acessá-las na sua íntegra, revelando-se um total de 17 produções.

Para auxiliar na organização e tabulação dos dados, foi utilizado programa de planilha eletrônica, sendo considerados os seguintes dados: tipo (Tese/dissertação), título, ano de publicação, instituição, estado de origem, referencial teórico, metodologia de pesquisa, (abordagem, coleta de dados e analise) e por fim, como o cuidado transcultural de Leininger foi abordado em cada produção. Para isto foram realizadas tabulações, comparando ano, tipo de produção e o item a ser analisado, totalizando em 08 tabelas com a representação das informações de cada produção.

Após terem sido extraídos os conteúdos contidos no instrumento de tabulação procederam-se os agrupamentos dos dados, considerando a similaridade dos mesmos. Desta forma, foi identificada a aplicação da Teoria da Diversidade e Universalidade do Cuidado Cultural de Madeleine Leininger em cada produção.

\section{RESULTADOS E DISCUSSÃO}

Pôde-se constatar que das 18 (dezoito) produções analisadas, 17 (dezessete) tratavam-se de dissertações de mestrado, enquanto que apenas 01 (uma) correspondeu a tese de doutorado. Assim, em relação aos anos de publicação, compreendidos entre 2005 e 2010, estas produções distribuíram-se em maior número no ano de 2006, com 06 (seis) ${ }^{5-10}$ dissertações de mestrado, seguido por 2007 com 05 (cinco) ${ }^{11-15}, 2009$ com 03 (três) ${ }^{16-18}$ e 2010 com 02 (duas) $^{19,20}$. A única tese de doutorado, por sua vez, foi concluída no ano de $2009^{21}$

Quanto às cidades, estados das regiões sul e sudeste, bem como as instituições das quais as produções se originaram, as dissertações e a tese, se encontravam assim distribuídas:

- Região Sudeste: Rio de Janeiro (RJ) - Universidade Estadual do Rio de Janeiro (UNIRIO) com 02 (duas) dissertações de mestrado ${ }^{16,17}$ e Universidade Federal do Rio de Janeiro (UFRJ) com 01 (uma) tese de doutorado $^{21}$; Belo Horizonte (MG) - Universidade Federal de Minas Gerais (UFMG) com 03 (três) dissertações de mestrado ${ }^{5,11,13}$; São Paulo (SP) Universidade de São Paulo (USP) com 01 (uma) dissertação de mestrado ${ }^{19}$.

- Região Sul: Florianópolis (SC) - Universidade Federal de Santa Catarina (UFSC) com 04 (quatro) dissertações de mestrado $7,8,18,19$; Curitiba (PR) Universidade Federal do Paraná (UFPR) com 03 (três) dissertações de mestrado ${ }^{9,10,12}$; Maringá (PR) Universidade Estadual de Maringá (UEM) com 02 (duas) dissertações de mestrado ${ }^{14,15}$; Rio Grande (RS) Universidade Federal de Rio Grande (FURG) com 01 (uma) dissertação de mestrado ${ }^{6}$.

Os tipos de pesquisa denominados nas dissertações de mestrado e tese de doutorado analisadas caracterizam-se, principalmente, como abordagem qualitativa, seguida da pesquisa convergente assistencial, etnográfica, descritiva e história de vida, como mostra a tabela 1.

Após a leitura dos trabalhos na íntegra, foi preenchida a tabela com os dados visando alcançar o objetivo desta pesquisa. As categorias que emergiram da análise dos dados coletados foram: Identificando a Teoria da Diversidade e Universalidade do Cuidado Cultural de Madeleine Leininger e $\mathrm{O}$ cuidado transcultural nas 
TABELA 1 - Distribuição das publicações, segundo a modalidade e tipo de pesquisa.

\begin{tabular}{lcc}
\hline \multicolumn{1}{c}{ Tipo de Pesquisa } & Dissertações de Mestrado & Tese de Doutorado \\
\hline Pesquisa Qualitativa, Convergente Assistencial & 06 & 05 \\
Pesquisa Qualitativa, Etnográfica & 02 \\
Pesquisa Qualitativa, Descritiva & 01 \\
Pesquisa Qualitativa & 01 \\
Pesquisa Qualitativa, Descritiva, Convergente Assistencial & 01 \\
Pesquisa Qualitativa, Exploratória & 01 & 01 \\
Pesquisa Qualitativa, História de Vida & 01 \\
\hline
\end{tabular}

pesquisas de enfermagem em saúde.

\section{Identificando a Teoria da Diversidade e Universalidade do Cuidado Cultural de Madeleine Leininger}

Entendendo que a transculturalidade vai além da apreciação das diferentes culturas, o enfermeiro deve estar capacitado para atender os seres humanos em sua totalidade, respeitando as diversidades culturais. Sendo importante considerar os aspectos culturais da necessidade humana para desenvolver um cuidado culturalmente congruente.

Madeleine Leininger descreve a Teoria da Diversidade e Universalidade do Cuidado Cultural como sendo:

[...] uma área maior da enfermagem que focaliza um estudo comparativo e analítico das diferentes culturas e subculturas no mundo, com respeito às condutas de cuidado dos enfermeiros, o cuidado de enfermagem, os valores do processo saúde-doença, as crenças e os padrões de comportamento com o objetivo de desenvolver um corpo de conhecimento científico e humanístico, visando prover uma cultura específica e uma prática de cuidado de cultura universal de enfermagem ${ }^{3: 39}$.

A teoria de Madeleine Leininger tem sido utilizada pelas pesquisadoras brasileiras há mais de trinta anos, como fundamentação para a prática assistencial, porém a utilização da análise tem sido pouco frequente. Ressaltam ainda que, as "produções mais amplas, envolvendo estudos de meta-análise ou de adequações e interpretações críticas de seu uso, na prática profissional, têm sido publicadas, mas não com a regularidade requerida $[\ldots]^{\prime 22: 221}$.
O referencial teórico de Madeleine Leininger foi utilizado em 10 trabalhos. As demais pesquisas associaram Madeleine Leininger a outros autores, tais como: Enrique Pichon-Riviere, Donald Winicot, Authur Kleinman e Wanda de Aguiar Horta. Percebe-se que nos trabalhos que foram baseadas em outros referenciais, a teoria de Madeleine Leininger foi utilizada para realização do trabalho de campo através da observação participante e entrevista etnográfica.

Leininger, pautada nas reflexões acerca da importância da cultura no cuidado de enfermagem, buscou outras áreas do conhecimento, fato este que explica o desenvolvimento do primeiro método de pesquisa genuinamente da enfermagem, denominando de Etnoenfermagem, que tem como definição:

[...] $\circ$ método de pesquisa qualitativa da enfermagem focalizada na abordagem naturalística, aberta à descoberta e amplamente indutiva para documentar, descrever, explicar e interpretar a visão de mundo, significados, símbolos e experiências de vida dos informantes e como eles enfrentam o atual ou potencial fenômeno do cuidado de enfermagem ${ }^{22}$.

Outro fator importante é que esta teoria foi desenvolvida a partir da prática vivenciada por Leininger com o objetivo de fundamentar a prática das enfermeiras, baseada na cultura e na aplicação de ações de enfermagem congruentes com crenças, valores, práticas, hábitos e costumes. A segunda abordagem mais utilizada está diretamente relacionada à teoria de Leininger, pois ela desenvolveu uma teoria e também uma metodologia para aplicar em outras pesquisas, nos diferentes contextos. 
O Cuidado Transcultural nas Práticas de Enfermagem em Saúde

Segundo Leininger, é indispensável conhecer e considerar os fatores para a compreensão do cuidado humanizado e, obter um conhecimento para um cuidado culturalmente congruente.

Os procedimentos utilizados para coleta de dados foram os preconizados pela pesquisa etnográfica, especialmente a entrevista, que esteve presente em todos os trabalhos. Leininger, em sua teoria, descreve o método O-P-R, ou seja, observação - participação reflexão. Também preconiza que seja utilizado um diário de campo e que as anotações sejam feitas enquanto o trabalho é realizado.

A entrevista, independente da forma, foi utilizada em todos os trabalhos analisados. A entrevista foi somada a outras técnicas e metodologias utilizadas para auxiliar o pesquisador a atingir os objetivos propostos na pesquisa, quais sejam: O-P-R, etnografia de James $P$. Spradley e história de vida.

As populações estudadas pela enfermagem nas produções foram cinco (5) com familiares e o mesmo número com pessoas da comunidade. Apenas duas (2) produções foram realizadas com a equipe de enfermagem e também o mesmo número, para as produções com pessoas atendidas em ambulatório e emergência no hospital. E, totalizando quatro (4) produções realizas com pessoas hospitalizadas.

O cuidado transcultural é uma teoria que procura abordar os aspectos da vida do ser humano, na sua complexidade, considerando as pessoas como seres de relações, fato este observado em um maior número de trabalhos realizados com pessoas na comunidade, assim como os que abordam as questões familiares.

Porém, também se identificou que a Teoria de Leininger pode ser aplicada em outros contextos de saúde, como o hospital, embora isto tenha sido representado por um pequeno número de produções (4) com pessoas hospitalizadas. No ambiente hospitalar, o modelo biomédico está mais fortemente presente, bem como é mais comum a utilização de outras formas de sistematização da assistência em enfermagem. Foram duas (2) as pesquisas com pessoas em atendimento no ambulatório e emergência, e totalizando dez (10) as pesquisas realizadas fora do ambiente hospitalar.

Os resultados sobre as estratégias de análise de dados utilizadas mostraram-se bem diversificadas, incluindo a teoria de Madeleine Leininger, a etnoenfermagem, etapas da pesquisa convergente assistencial, discurso do sujeito coletivo, análise de conteúdo e temática, como podemos verificar na tabela 2.

Após a leitura das produções inclusas no estudo, estas foram analisadas quanto à utilização da teoria da Diversidade e Universalidade do Cuidado Cultural de Madeleine Leininger. As categorias utilizadas para os resultados foram estruturadas após a leitura, conforme o que foi surgindo nos trabalhos.

Desta forma, a teoria foi assim utilizada: referencial teórico (10), metodologia para coleta de dados (10); análise dos dados (8), modelo do Sol Nascente (5); as ações de preconizadas por Leininger - manutenção, acomodação e repadronização (5). Através destes dados pode-se concluir que os pesquisadores não utilizaram os conceitos de Leininger em sua totalidade na pesquisa, ou seja, utilizaram partes da teoria, conforme os objetivos de seus trabalhos.

Sendo assim, em nenhuma das produções o Modelo do Sol Nascente foi utilizado na íntegra. Alguns utilizaram apenas o primeiro nível, outros apenas os conceitos e outros, as ações integradas aos conceitos.

O Modelo do Sol Nascente traz componentes básicos, que simbolizam, através do nascer do sol, as formas de descobrir o cuidado num entendimento da visão de mundo, das estruturas sociais e fatores variáveis como: tecnológico; religioso e filosófico; familiares; maneiras de viver segundo crenças e valores; políticos e legais; econômicos e educacionais ${ }^{3}$.

Madeleine Leininger desenvolveu uma metodologia em que a sua teoria pode ser utilizada na prática das ações de cuidado. Assim, define que as ações de cuidado, têm o intuito de auxiliar e direcionar as ações e decisões de enfermagem, quais sejam: manutenção ou preservação do cuidado cultural, acomodação ou negociação do cuidado cultural e reestruturação ou repadronização do cuidado cultural ${ }^{3}$.

A manutenção ou preservação do cuidado cultural está direcionada às ações ou decisões profissionais que 
TABELA 2 - Distribuição das publicações, segundo a análise dos dados.

\begin{tabular}{|c|c|c|c|}
\hline Método de Análise dos Dados & Dissertações de Mestrado & Tese de Doutorado & Total Parcial \\
\hline Teoria de Madeleine Leininger & 06 & & 06 \\
\hline Etnoenfermagem de Madeleine Leininger & 02 & & 02 \\
\hline Etnografia de James P. Spradley & 03 & & 03 \\
\hline Etapas da Pesquisa Convergente Assistencial & 02 & & 02 \\
\hline Análise de Conteúdo de Bardin & 03 & & 03 \\
\hline Análise Temática & & 01 & 01 \\
\hline Análise do Discurso do Sujeito Coletivo & 02 & & 02 \\
\hline
\end{tabular}

conservam ou preservam a cultura, frente às diferenças em relação ao sistema profissional de cuidado. Já a acomodação ou negociação do cuidado cultural, referese às ações ou decisões profissionais que fortalecem os indivíduos e suas famílias para adaptar as práticas de cuidado popular, evitando a imposição cultural. $\mathrm{Na}$ reestruturação ou repadronização do cuidado cultural, o profissional auxilia o sistema popular a modificar seus estilos de vida, em prol da organização familiar ${ }^{3}$.

A identificação da utilização da teoria de Madeleine Leininger nas produções científicas dos programas de Pós-Graduação em Enfermagem na região sul e sudeste do Brasil permitiu-nos perceber que a teoria não é utilizada de maneira uniforme, ou seja, alguns autores a utilizam de forma fragmentada, porém, em outras produções, esta é utilizada em todo o trabalho, mas apenas parte da teoria.

As propostas metodológicas utilizadas nos achados das produções científicas foram flexíveis e criativas, utilizando-se diversas técnicas e instrumentos para a implementação do cuidado cultural.

Porém, foi possível afirmar de que precisamos aprimorar as pesquisas acerca do cuidado transcultural, acreditando ser necessário repensar o entendimento do que é o cuidado, como ele deve ser prestado e qual o olhar dado pelas equipes de enfermagem neste âmbito.

A utilização de teorias para nortear a assistência de enfermagem permite que esta seja diferenciada e com embasamento científico, prestando um cuidado diferenciado do cuidado cotidiano. Incluir no planejamento da assistência de enfermagem os conhecimentos prévios de pessoas a serem cuidadas e suas famílias permite maior aproximação das equipes de enfermagem à comunidade, com vistas numa assistência mais efetiva e preocupada com as atitudes, crenças e valores das pessoas que buscam os cuidados para atender suas necessidades dentro do processo de saúde-doença.

\section{REFERÊNCIAS}

1. Waldov VR. Cuidar expressão humanizadora da enfermagem. Petrópolis: Vozes; 2006. 190p.

2. Leopardi MT. Teoria e método em assistência de enfermagem. Florianópolis: Soldasoft; 2006. 396p.

3. Leininger MM. Culture care diversity and university: a theory of nursing. New York: National League for Nursing Press; 1991. 432p.

4. Ganong LH. Integrative reviews of nursing research. Res Nurs Health. 1987 Feb;10(1):1-11

5. Almeida SP de. A vivência no grupo: a experiência para as pessoas diabéticas [dissertação]. Belo Horizonte (MG): UFMG, Escola de Enfermagem; 2006.

6. Bulhosa MS. Sofrimento moral no trabalho da enfermagem [dissertação]. Rio Grande (RS): FURG, Enfermagem; 2006.

7. Portela CM. O cuidado ao familiar cuidador de portadores de transtornos de humor na rede básica de atenção à saúde [dissertação]. Florianópolis (SC) UFSC/PEN; 2006.

8. Saraiva MC. Assistência de saúde a idosos que buscam atendimento quiroprático [dissertação]. Florianópolis (SC) UFSC, PEN; 2006

9. Modesto AP. O cuidado cultural do idoso renal crônico em tratamento hemodialítico [dissertação]. Curitiba (PR): UFPR/Enfermagem; 2006

10. Gerondo VL dos S. As avós idosas cuidadoras dos netos hospitalizados [dissertação]. Curitiba (PR): UFPR, Enfermagem; 2006.

11. Fernandes MT de $O$. Trabalho com grupos na saúde da família: concepções, estrutura e estratégias para o cuidado transcultural [dissertação]. Belo Horizonte (MG): UFMG, Escola de Enfermagem; 2007.

12. Kuznier TP. O significado do envelhecimento e do cuidado para o idoso hospitalizado e as possibilidades do cuidado de si [dissertação]. Curitiba (PR): UFPR, Enfermagem; 2007.

13. Santos CA dos. Gravidez e soropositividade para o HIV: vivências de mulheres atendidas em um centro de referência em HIV/aids [dissertação]. Belo Horizonte (MG): UFMG, Escola de Enfermagem; 2007. 
14. Tomeleri KR. Experiência de mães adolescentes cuidando de seus filhos nos primeiros seis meses de vida (A) [dissertação]. Maringá (PR): UEM, Enfermagem; 2007.

15. Molina RCM. Inserção da mãe no cuidado ao filho internado na unidade de terapia intensiva pediátrica [dissertação]. Maringá (PR): UEM, Enfermagem; 2007.

16. Rosa JG da. Mãe $x$ mulher: a abstinência sexual na ameaça de parto prematuro na perspectiva sociocultural [dissertação]. Rio de Janeiro (RJ): UNIRIO, Enfermagem; 2009.

17. Silva RF da. Valores culturais que envolvem o cuidado materno ribeirinho: subsídios para a enfermagem [dissertação]. Rio de Janeiro (RJ): UNIRIO, Enfermagem; 2009.

18. Souza AC. O itinerário terapêutico das famílias de crianças com diarréia [dissertação]. Florianópolis (SC): UFSC, PEN; 2009.

19. Ferraz L. Cuidado prestado à criança pela família e comunidade indígena na percepção dos profissionais de saúde (O) [dissertação]. Florianópolis (SC): UFSC/PEN, 2010
20. Bonini BB. Ser enfermeiro negro na perspectiva da transculturalidade do cuidado [dissertação]. São Paulo (SP): USP, Escola de Enfermagem; 2010.

21. Santos IMM dos. Maternagem de mulheres com filho prétermo: bases para assistência de enfermagem neonatal (A) [tese]. Rio de Janeiro (RJ): UFRJ; Escola de Enfermagem Anna Nery; 2009.

22. Monticelli M, Boehs AE, Guesser JC, Gehrmann T, Martins M, Manfrini GC. Aplicações da teoria transcultura na prática da enfermagem a partir de dissertações de mestrado. Texto Contexto Enferm. [periódico online]. 2010 Apr/June; 19(2): 220-8. [capturado 2011 Dec 07] Disponível em: http://www.scielo.br/pdf/tce/v19n2/02.pdf.

\section{Endereço para correspondência:}

Luizita Henckemaie

Rua Prefeito Dib Cherem n²881, Apto. 302

Florianópolis/SC - CEP 88090000

Telefone: +55 4891618396 / +55 4832497059

E-mail: luizitahe@gmail.com 